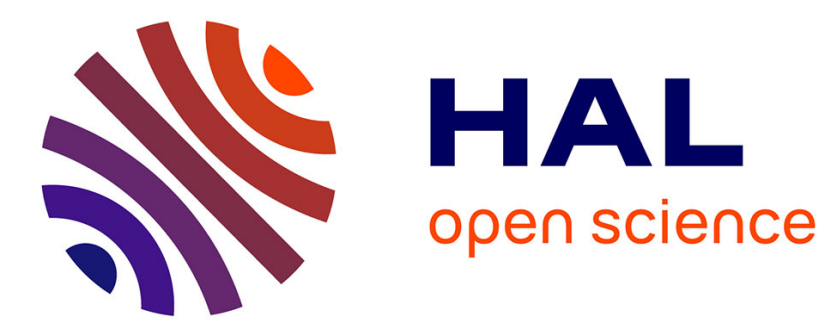

\title{
Modelés et formations quaternaires du terrefort molassique, au Sud de Toulouse
}

Jacques Hubschman

\section{To cite this version:}

Jacques Hubschman. Modelés et formations quaternaires du terrefort molassique, au Sud de Toulouse. Bulletin de l'Association française pour l'étude du quaternaire, 1975, 12 (3-4), pp.125136. 10.3406/quate.1975.1260 . hal-02736251

\section{HAL Id: hal-02736251 \\ https://hal-univ-tlse2.archives-ouvertes.fr/hal-02736251}

Submitted on 2 Jun 2020

HAL is a multi-disciplinary open access archive for the deposit and dissemination of scientific research documents, whether they are published or not. The documents may come from teaching and research institutions in France or abroad, or from public or private research centers.
L'archive ouverte pluridisciplinaire HAL, est destinée au dépôt et à la diffusion de documents scientifiques de niveau recherche, publiés ou non, émanant des établissements d'enseignement et de recherche français ou étrangers, des laboratoires publics ou privés. 


\section{I. - Modelés et formations quaternaires du terrefort molassique, au}

\section{Sud de Toulouse}

\section{Jacques Hubschman}

\section{Abstract}

In the hills of the molassic region, high surfaces surround recent colluvium covered slopes and little loess deposits in hollows. Soil profiles of hight surfaces are relatively young : hight surfaces have been recently shapened (Würm I ? ). One big loess profile is described and analyzed (second part of Würm).

\section{Citer ce document / Cite this document :}

Hubschman Jacques. I. - Modelés et formations quaternaires du terrefort molassique, au Sud de Toulouse. In: Bulletin de I'Association française pour l'étude du quaternaire, vol. 12, n³-4, 1975. pp. 125-136;

doi : https://doi.org/10.3406/quate.1975.1260

https://www.persee.fr/doc/quate_0004-5500_1975_num_12_3_1260

Fichier pdf généré le 19/04/2018 


\section{TERREFORT MOLASSIQUE ET TERRASSES RÉCENTES DE LA RÉGION TOULOUSAINE}

La matinée de cette première journée a été consacrée à la visite des installations du Laboratoire de Géochronologie de l'Université Paul-Sabatier de Toulouse. Un séminaire, organisé par M. Yves Gourinard a porté sur la méthode Potassium/Argon et les problèmes qu'elle pose. Les principaux aspects en ont été présentés dans un précédent numéro du Bulletin.

Le programme de l'après-midi s'est déroulé d'abord dans les coteaux molassiques du Terrefort (entre Garonne et Hers mort) et sur les formations récentes de la plaine garonnaise. Cette première journée s'achevait sur une brève visite de la galerie de Préhistoire du Muséum d'Histoire Naturelle de Toulouse, visite commentée par M.J. Clottes, Directeur des Antiquités préhistoriques de Midi-Pyrénées.

\section{I. - Modelés et formations quaternaires du terrefort molassique, au sud de Toulouse}

par Jacques HUBSCHMAN*

\section{SUMMARY}

In the hills of the molassic region, high surfaces surround recent colluvium covered slopes and little loess deposits in hollows. Soil profiles of hight surfaces are relatively young: hight surfaces have been recently shapened (Würm I?). One big loess profile is described and analyzed (second part of Würm).

\section{1. - L'ORGANISATION GEOMORPHOLOGIQUE}

$\mathrm{Au}$ sud de Toulouse, entre l'Ariège-Garonne et la vallée de l'Hers mort, la molasse d'âge stampien qui forme les coteaux offre une large dominance de matériaux meubles argilo-calcaires, localement traversés de lentilles sableuses. Les bancs calcaires ou gréso-calcaires qui, ailleurs, arment la molasse, sont en effet très rares ici et les formes structurales qu'ils imposent pratiquement inexistantes ou aisément démasquées.

(*) Laboratoire de Géographie Physique, Université de Toulouse-Le Mirail, 109 bis rue Vauquelin, 31081 Toulouse Cédex. 


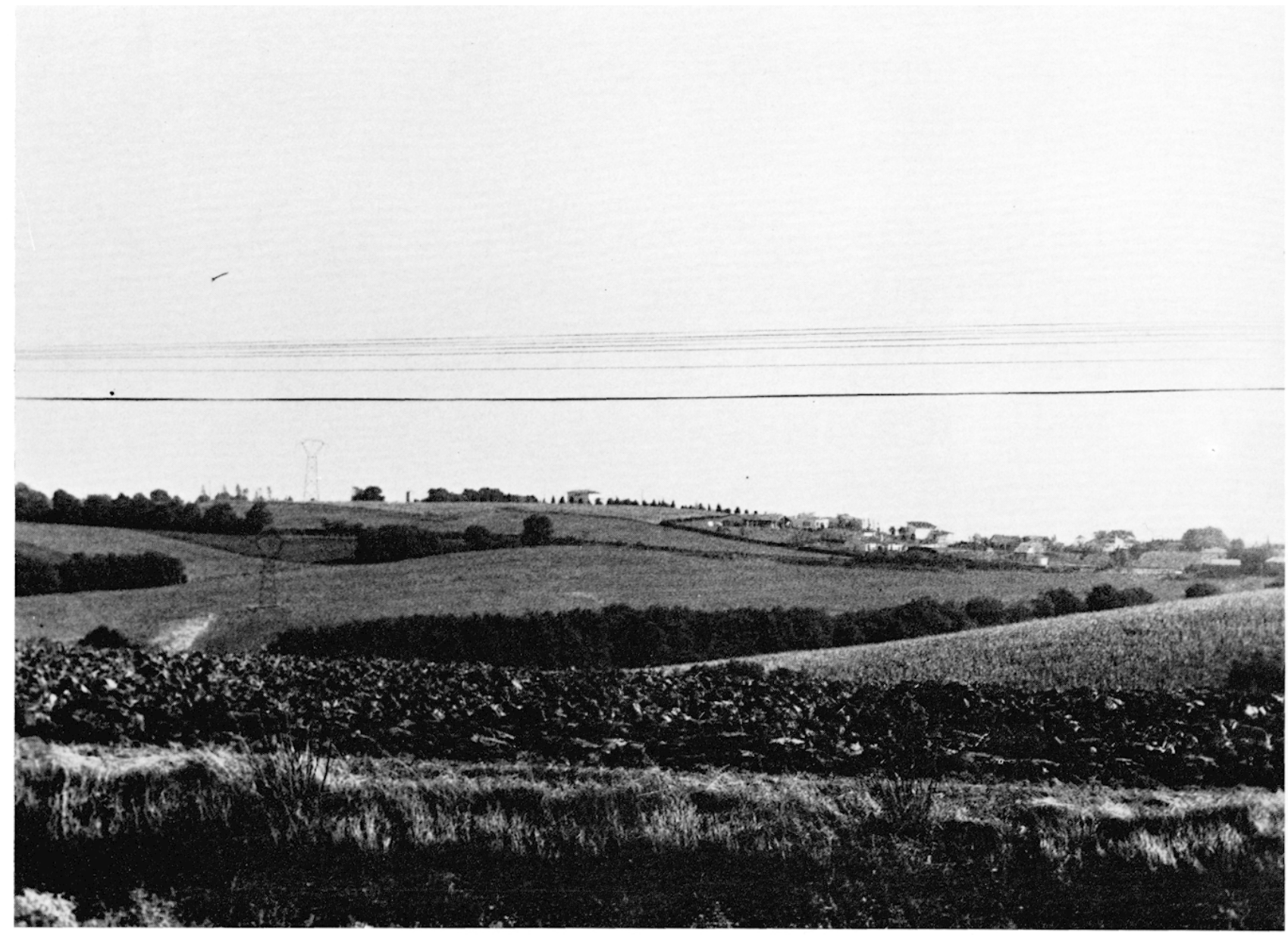

Photo 1 - Le terrefort molassique lauragais.

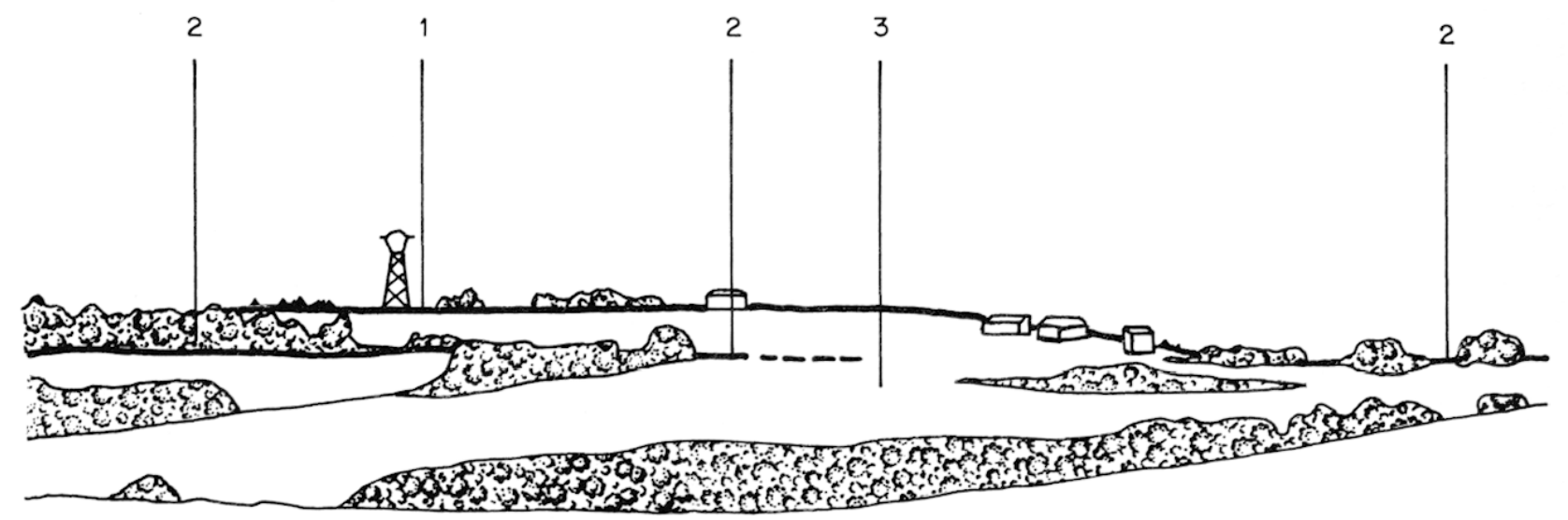

Fig. 2 - L'organisation géomorphologique du Terrefort molassique lauragais (cf. photo 1). 1 : replats supérieurs ; 2 : replats inférieurs ; 3 : versants longs à pente modérée.

Un dense réseau de petits cours d'eau a disséqué cette région en un fouillis de molles collines et de vallons encaissés. Ce modelé complexe obéit cependant à un certain ordre et les morphosculptures, bien visibles depuis l'arrêt $n^{\circ} 3$, s'organisent de la façon suivante (photo 1 et figure 2) :

- des éléments de plateau, généralement allongés sous forme de longues et étroites échines (quelques dizaines à une centaine de mètres de largeur dans la plupart des cas), à la surface légèrement ondulée, faiblement inclinées du SE au NW, vers $260-280 \mathrm{~m}$ d'altitude. Ces échines passent par endroit à des niveaux inférieurs, plus ou moins clairement emboîtés sous elles, vers $230-250 \mathrm{~m}$, souvent un peu plus larges et qui constituent également un long système d'interfluves entre les vallons ; 
- un système de versants longs, à pente modérée, assez fréquemment tournés vers l'est ou le NE (légère dissymétrie des vallons), inscrits sous les niveaux précédents et auxquels font face les pentes raides des versants courts où affleure parfois la molasse en place.

A l'ouest, ce secteur du Terrefort domine la Garonne et l'Ariège par un raide et haut talus d'une centaine de mètres de commandement. A l'est, en revanche, le versant qui regarde l'Hers mort est bien plus doux; par endroit, il est jalonné par quelques replats, lambeaux de terrasses alluviales de l'Hers mort, que l'on peut observer de l'église de Pompertuzat (au-dessus de l'arrêt $n^{\circ} 4$ ).

\section{II. - LES FORMATIONS QUATERNAIRES ET LES SOLS}

\section{A - Le manteau colluvial du Terrefort}

Les versants longs du Terrefort sont, de façon très générale, recouverts d'un manteau colluvial dont le matériel provient, dans une large mesure, de la destruction partielle des éléments de plateau dominants. Ce manteau, qui ravine la molasse, est plus ou moins continu et d'épaisseur variable : de l'ordre de quelques décimètres au sommet des versants, il peut atteindre un et parfois plusieurs mètres à leur pied ; cette formation passe alors latéralement aux remblaiements souvent épais qui colmatent les fonds de vallons. Ces dépôts sont généralement fins, limono-argileux : c'est "l'argile de coulière" de G. Astre (1953 a, 1959). Mais ils revêtent parfois une allure plus grossière, notamment sur le haut des versants: s'y mêlent alors des débris de molasse ou des fragments d'horizons d'accumulation calcaire des sols de plateau.

Précisément, l'arrêt $\mathrm{n}^{\circ} 1$, à la sortie de RamonvilleSaint-Agne (D. 35), permet de saisir en quelque sorte la "racine" du limon colluvial. Le "départ" se fait à partir d'une croupe molassique secondaire dominant le vallon de Langlade. Au sommet de la croupe, la maigre rendzine sur molasse passe latéralement à une formation encore mince, mi-colluviale, mi-éluviale, à rendzine brunifiée, qui s'épanouit vers le bas en un revêtement plus épais à profil de sol brun calcique. On note au passage certains des faciès typiques de la série molassique stampienne : faciès argilo-calcaire à rognons gréso-calcaires consolidés; bariolage à teintes gris-bleu et rouille dominantes, traduisant la redistribution du fer liée aux conditions hydromorphes qui prévalaient au Stampien.

Ce type de formation colluviale s'observe à nouveau à l'arrêt $\mathrm{n}^{\circ} 2$, situé au pied du long versant de Pechbusque, dans le vallon de Langlade. Le dépôt est ici plus épais ( $1 \mathrm{~m}$ environ) mais, au lieu de recouvrir directement la molasse, il coiffe et ravine un petit placage loessique. Le loess, en effet, tapisse très localement quelques fonds de vallon à l'intérieur même du Terrefort: cependant, nous verrons plus loin que les principales accumulations éoliennes caractérisent surtout le versant gauche de la vallée de l'Hers mort (arrêt $\left.n^{\circ} 5\right)$.

\section{Description sommaire du profil de l'arrêt $n^{\circ} 2$ :}

sol brun calcique (à profil textural légèrement différencié) :

- 0-30 cm: Ap, 10 YR 4/4 à 2,5 YR 5/4, structure massive, sous-structure grumeleuse, moyennement cohérent. Fragments de briques ou tuiles. Racines, cavités zoogènes. Limite inférieure ondulée, passage assez net à (B) ;

- 30-110 cm: (B) et IC, 10 YR 5/4 à 2,5 YR 6/4, structure polyédrique moyenne à forte, plus massive en profondeur, faces légèrement lissées. Cohésion moyenne. Limite inférieure très nette, par ravinement, avec II C ;

- 110-195 cm: II C, loess, 2,5 Y 7/4, structure massive, faiblement cohérent. Pseudomycélium, vermicules, tubes racinaires et nodules calcaires pourris.

\begin{tabular}{|c|c|c|c|c|c|c|}
\hline \multirow{3}{*}{$\begin{array}{c}\text { Prof. } \\
\mathrm{cm}\end{array}$} & \multicolumn{6}{|c|}{ G:- 'ulométrie \% } \\
\hline & \multirow[b]{2}{*}{ EG } & \multicolumn{5}{|c|}{$\%$ de TF } \\
\hline & & SG & SF & STF & $\mathrm{L}$ & A \\
\hline $10-20$ & 0 & 3,2 & 19,5 & 27,3 & 16,2 & 31,3 \\
\hline $40-50$ & 0 & 0,9 & 18,5 & 26,4 & 16,4 & 36,8 \\
\hline $70-80$ & 0 & 0,4 & 16,7 & 26,0 & 16,9 & 38,7 \\
\hline $130-140$ & 0 & 7,2 & 32,3 & 31,0 & 22,9 & 6,3 \\
\hline
\end{tabular}

\begin{tabular}{|c|c|c|c|c|c|c|c|c|}
\hline \multirow{2}{*}{$\begin{array}{c}\text { Prof. } \\
\mathrm{cm}\end{array}$} & \multicolumn{2}{|c|}{ Calcaire \% } & \multicolumn{2}{|c|}{$\mathrm{pH}$} & \multicolumn{4}{c|}{ Matière organique \% } \\
\cline { 2 - 10 } & $\mathrm{T}$ & $\mathrm{A}$ & $\mathrm{eau}$ & $\mathrm{KCl}$ & $\mathrm{C}$ & $\mathrm{MO}$ & $\mathrm{N}$ & $\mathrm{C} / \mathrm{N}$ \\
\hline $10-20$ & 0,8 & 0 & 7,7 & 6,8 & 0,88 & 1,5 & 0,13 & 6,8 \\
$40-50$ & 0,3 & 0 & 7,1 & 0,6 & 0,36 & 0,6 & 0,07 & 5,0 \\
$70-80$ & 0,7 & 0 & 8,0 & 0,5 & 0,5 & 0,30 & 0,06 & 4,4 \\
$130-140$ & 28,3 & 6,3 & 8,0 & 0,2 & 0,2 & 0,13 & 0,02 & 4,8 \\
\hline
\end{tabular}




\begin{tabular}{|c|c|c|c|c|c|c|c|}
\hline \multirow{2}{*}{$\begin{array}{c}\text { Prof. } \\
\mathrm{cm}\end{array}$} & \multicolumn{8}{|c|}{ Complexe absorbant meq \% } & \multicolumn{2}{c|}{$\mathrm{T}$} & $\mathrm{S} / \mathrm{T} \%$ \\
\cline { 2 - 8 } & $\mathrm{Ca}$ & $\mathrm{K}$ & $\mathrm{Mg}$ & $\mathrm{Na}$ & $\mathrm{S}$ & \multicolumn{2}{c|}{$\mathrm{T}$} \\
\hline $10-20$ & 30,8 & 0,32 & 1,5 & 0,13 & 32,8 & 20,0 & sat. \\
$40-50$ & 24,3 & 0,28 & 1,3 & 0,10 & 25,7 & 19,9 & sat. \\
$70-80$ & 34,6 & 0,30 & 1,5 & 0,13 & 36,5 & 24,6 & sat. \\
$130-140$ & 47,9 & 0,13 & 1,4 & 0,13 & 49,5 & 6,1 & sat. \\
\hline
\end{tabular}

\begin{tabular}{|c|c|c|c|c|c|c|c|}
\hline $\begin{array}{c}\text { Prof. } \\
\mathrm{cm}\end{array}$ & Illites & Chlorite & Vermiculite & $\begin{array}{c}\text { Montmo- } \\
\text { rillonite }\end{array}$ & Kaolinites & $\begin{array}{c}\text { Inter. } \\
10-14 \\
14 \mathrm{C}-14 \mathrm{~V}\end{array}$ & $\begin{array}{c}\text { Interstr. } \\
\text { gonflants }\end{array}$ \\
\hline $10-20$ & $\mathrm{~m}$ & $\mathrm{~m}$ & $\mathrm{f}$ & $\mathrm{M}$ & $\mathrm{f}$ & $\mathrm{m}$ & $\mathrm{m}$ \\
$70-80$ & $\mathrm{~m}$ & $\mathrm{tr}$ & $\mathrm{f}$ & $\mathrm{m}$ & $\mathrm{f}$ & $\mathrm{m}$ & $\mathrm{f}$ \\
$130-140$ & $\mathrm{~m}$ & $\mathrm{f}$ & $\mathrm{m}$ & $\mathrm{F}$ & $\mathrm{f}$ & & $\mathrm{m}$ \\
\hline
\end{tabular}

$F$ : fort ; $M$; moyen fort ; $m:$ moyen faible ; $f$ faible ; $t r:$ traces Abréviations ou symboles des tableaux d'analyse :

EG : éléments grossiers supérieurs à $2 \mathrm{~mm}$; TF : terre fine ; SG, SF, STF : sables grossiers, fins, très fins ; L : limons ; A : argiles.

Calcaire T, A : total, actif ; MO : matière organique ; meq : milli-équivalent ; $\mathrm{S}$ : somme des bases échangeables ; $\mathrm{T}:$ capacité d'échange.

On notera :

- la légère différenciation du profil textural, plus marquée que de coutûme dans les sols bruns calciques. Elle semble traduire surtout le fort remaniement de Ap, horizon superficiel de bas de pente, engraissé par la mise en culture du versant (fragments de briques fréquents), d'autant que les lames minces ne montrent pas trace de cutanes argileux en (B);

- la relative abondance de minéraux gonflants, montmorillonite y compris (surtout dans le loess). De là sans doute l'allure faiblement vertique du profil structural (éléments à faces légèrement lissées, brillantes, discrètement conchoïdales). Rappelons à cet égard que, dans les séries molassiques du Bassin Aquitain, les smectites jouent un rôle important, en dépit d'une distribution assez irrégulière. L'abondance de ces minéraux semble en relation avec l'existence de minces paléosols hydromorphes et vertiques, empilés dans les formations molassiques miocènes et oligocènes et dans lesquels les smectites se seraient néoformées (F. Crouzel et al., 1972).

L'âge de ces dépôts de pente à sols peu évolués est postérieur à la mise en place du loess sous-jacent. Celle-ci remonte, selon toute vraisemblance, à la fin du Würm (arrêt $n^{\circ}$ 5). L'établissement du manteau colluvial date donc probablement et pour l'essentiel de l'extrême fin du Würm ou des premières phases du Postglaciaire, avant l'installation de la grande chênaie. Les derniers stades d'engraissement résultent des défrichements historiques, médiévaux et actuels (1).

(1) Il faut noter que, localement, l'épais matériel qui colmate le fond de certains vallons du Terrefort a livré un outillage et une faune qui trahissent un remblaiement plus ancien, probablement du début du Würm (L. Méroc et J. Paloumé 1958).

\section{B - L'évolution des éléments de plateau}

De l'arrêt $\mathrm{n}^{\circ} 3$, à $270 \mathrm{~m}$ et au-dessus de Pechbusque (D 35), on domine l'ensemble de ce secteur molassique. Les longues crêtes d'interfluve qui barrent l'horizon rappellent le vaste plateau molassique originel, faiblement incliné du SE au NW, aujourd'hui vigoureusement défoncé par un système complexe de vallons (photo 2). Ces hauts niveaux représentent-ils des témoins de surfaces anciennes, comme cela a pu être suggéré (G. Astre 1953 b, H. Enjalbert 1960, A. Cavaillé 1969 a) ?

En fait, si les éléments de plateau qui dominent aujourd'hui dans le paysage ont sans doute partiellement hérité le dessin général de la haute surface molassique initiale, ils n'en ont conservé ni l'altitude, ni le matériel molassique superficiel. L'évolution pédologique de ces replats demeure en effet presque toujours peu poussée, ce qui postule un énergique rajeunissement de ces surfaces depuis la fin du Tertiaire. Le profil étudié en donne un exemple (photo 3 ).

\section{Description sommaire du profil de l'arrêt $n^{\circ} 3$ :}

sol brun lessivé à horizon d'accumulation calcaire :

- 0-20 cm: Ap, 10 YR 5/4, structure massive, sous-structure grenue puis polyédrique fine en profondeur, racines, forte activité biologique, passage progressif à $\mathrm{A}_{2}$, limite ondulée ;

- 20-50 cm: $A_{2}, 10$ YR 4/4, structure massive à tendance lamellaire puis polyédrique, fendillé, passage progressif à $B_{1 t}$, limite faiblement ondulée ;

- 50-80 cm: $B_{1 t}, 10$ YR 4/3 à 7,5 YR 4/4, structure polyédrique moyenne à tendance prismatique, faces légèrement lissées, revêtements argileux, fendillé, passage progressif à $\left.\mathrm{B}_{2 \mathrm{t}}(\mathrm{g}) \mathrm{(}^{*}\right)$; 
$-80-95 \mathrm{~cm}: \mathrm{B}_{2 \mathrm{t}(\mathrm{g})}$ à $\mathrm{B}_{\mathrm{c}(\mathrm{g})}$, crème, plages grises et rouilles de la molasse, tendance tuffeuse locale, quelques concrétions dures et creuses à cortex pourri, passage progressif à $\mathrm{C}_{(\mathrm{g})}$;
- 110-195 cm: $C_{(g)}$, molasse stampienne, plages grises et rouilles en taches ou en lits, minces lits de calcaire pulvérulent sub-horizontaux.

(g)* caractère pseudogley hérité de la molasse.

\begin{tabular}{|c|c|c|c|c|c|c|}
\hline \multirow{3}{*}{$\begin{array}{l}\text { Prof. } \\
\mathrm{cm}\end{array}$} & \multicolumn{6}{|c|}{ Granulométrie \% } \\
\hline & \multirow[b]{2}{*}{ EG } & \multicolumn{5}{|c|}{$\%$ de TF } \\
\hline & & SG & SF & STF & $L$ & A \\
\hline $10-20$ & 0 & 20,0 & 22,4 & 14,0 & 16,8 & 23,3 \\
\hline $35-45$ & 0 & 14,0 & 19,7 & 15,5 & 19,6 & 29,3 \\
\hline $70-80$ & 0 & 7,8 & 13,6 & 12,7 & 23,0 & 41,4 \\
\hline $100-110$ & 8,4 & 5,6 & 23,8 & 17,8 & 28,7 & 23,6 \\
\hline $140-150$ & 0 & 10,1 & 25,6 & 13,2 & 27,9 & 22,2 \\
\hline
\end{tabular}

\begin{tabular}{|c|c|c|c|c|c|c|c|c|}
\hline \multirow{2}{*}{$\begin{array}{c}\text { Prof. } \\
\text { cm }\end{array}$} & \multicolumn{2}{|c|}{ Calcaire \% } & \multicolumn{3}{|c|}{$\mathrm{pH}$} & \multicolumn{3}{c|}{ Matière organique \% } \\
\cline { 2 - 9 } & $\mathrm{T}$ & $\mathrm{A}$ & eau & KCl & MO & $\mathrm{C}$ & $\mathrm{N}$ & $\mathrm{C} / \mathrm{N}$ \\
\hline $10-20$ & 2,7 & 0 & 8,3 & 7,2 & 0,7 & 0,45 & 0,08 & 5,6 \\
$35-45$ & 1,0 & 0 & 8,4 & 7,1 & 0,9 & 0,54 & 0,05 & 9,5 \\
$70-80$ & 0,8 & 0 & 8,3 & 7,0 & 0,6 & 0,39 & 0,05 & 7,5 \\
$100-110$ & 16,9 & 4,6 & 8,4 & 7,2 & 0,4 & 0,24 & 0,03 & 6,2 \\
$140-150$ & 12,1 & 2,9 & 8,4 & 7,3 & & & & \\
\hline
\end{tabular}

\begin{tabular}{|c|c|c|c|c|c|c|c|c|c|c|}
\hline \multirow{2}{*}{$\begin{array}{c}\text { Prof. } \\
\mathrm{cm}\end{array}$} & \multicolumn{9}{|c|}{ Complexe absorbant meq \% } & \multicolumn{3}{|c|}{ Fe \% } \\
\cline { 2 - 11 } & & $\mathrm{K}$ & $\mathrm{Mg}$ & $\mathrm{Na}$ & $\mathrm{S}$ & $\mathrm{T}$ & $\mathrm{S} / \mathrm{T} \%$ & $\mathrm{FT}$ & $\mathrm{FL}$ & $\frac{\mathrm{FL}}{\mathrm{FT}}$ \\
\hline $10-20$ & 35,4 & 0,21 & 1,1 & 0,07 & 36,7 & 14,5 & sat. & 2,24 & 1,52 & $\mathrm{t}$ \\
$35-45$ & 26,4 & 0,21 & 1,3 & 0,07 & 27,9 & 15,4 & sat. & 2,36 & 1,45 & 61 \\
$70-80$ & 31,1 & 0,32 & 1,3 & 0,13 & 32,8 & 17,7 & sat. & 3,18 & 1,96 & 61 \\
$100-110$ & 42,9 & 0,28 & 1,2 & 0,13 & 44,5 & 19,9 & sat. & 2,93 & 1,87 & 63 \\
\hline
\end{tabular}

\begin{tabular}{|c|c|c|c|c|c|c|c|}
\hline $\begin{array}{c}\text { Prof. } \\
\mathrm{cm}\end{array}$ & Illites & Chlorite & Vermiculite & $\begin{array}{c}\text { Montmo- } \\
\text { rillonite }\end{array}$ & Kaolinites & $\begin{array}{c}\text { Inter. } \\
10-14 \\
14 \mathrm{C}-14 \mathrm{~V}\end{array}$ & $\begin{array}{c}\text { Interstr. } \\
\text { gonflants }\end{array}$ \\
\hline $10-20$ & $\mathrm{M}$ & $\mathrm{tr}$ & $\mathrm{f}$ & $\mathrm{m}$ & $\mathrm{f}$ & $\mathrm{M}$ & $\mathrm{f}$ \\
$70-80$ & $\mathrm{~m}$ & $\mathrm{f}$ & $\mathrm{m}$ & $\mathrm{M}$ & $\mathrm{m}$ & $\mathrm{m}$ & $\mathrm{m}$ \\
$100-110$ & $\mathrm{f}$ & $\mathrm{f}$ & $\mathrm{f}$ & $\mathrm{F}$ & $\mathrm{m}$ & $\mathrm{m}$ & $\mathrm{tr}$ \\
$140-150$ & $\mathrm{~m}$ & $\mathrm{~m}$ & $\mathrm{~m}$ & $\mathrm{~F}$ & $\mathrm{~m}$ & $\mathrm{tr}$ & $\mathrm{f}$ \\
\hline
\end{tabular}

$\mathrm{F}:$ fort $; \mathbf{M}:$ moyen fort $; \mathrm{m}:$ moyen faible $; \mathbf{f}:$ faible $; \mathrm{tr}:$ traces.

Abréviations : FT, FL : fer total, fer libre (Deb). Pour les autres abréviations, voir tableau précédent.

- Malgré une différenciation déjà nettement exprimée, ce type de sol reste encore relativement "jeune". Même si l'on tient compte de la différence de rochemère, de site géomorphologique et de conditions hydrodynamiques, il est clair que ces "boulbènes de plateau" sont bien éloignées des profils franchement lessivés hydromorphes développés dans les terrasses rissiennes et antérissiennes de la Garonne (arrêts ${ }^{\circ} 7$ et 8 et $2^{\mathrm{e}}$ journée).

- D'autre part et surtout, le cortège de minéraux argileux du sol ne traduit qu'une évolution ménagée des phyllites de la molasse. Ainsi, la montmorillonite reste abondante dans les $B_{t}$ et ne s'estompe nettement que dans les horizons supérieurs du profil. On imagine mal qu'il puisse en être ainsi dans un sol dont le développement se serait amorcé à une période réellement ancienne. De fait, dans tous les profils des terrasses antéwürmiennes (et, bien souvent, würmiennes) de la Garonne, plus acides il est vrai, les montmorillonites de la molasse ont totalement disparu.

- L'horizon d'accumulation du calcaire, $\mathrm{C}_{\mathrm{Ca}(\mathrm{g})}$ ou $\mathrm{B}_{\mathrm{Ca}(\mathrm{g})}$, associe à la fois des faciès diffus ou pulvé- 


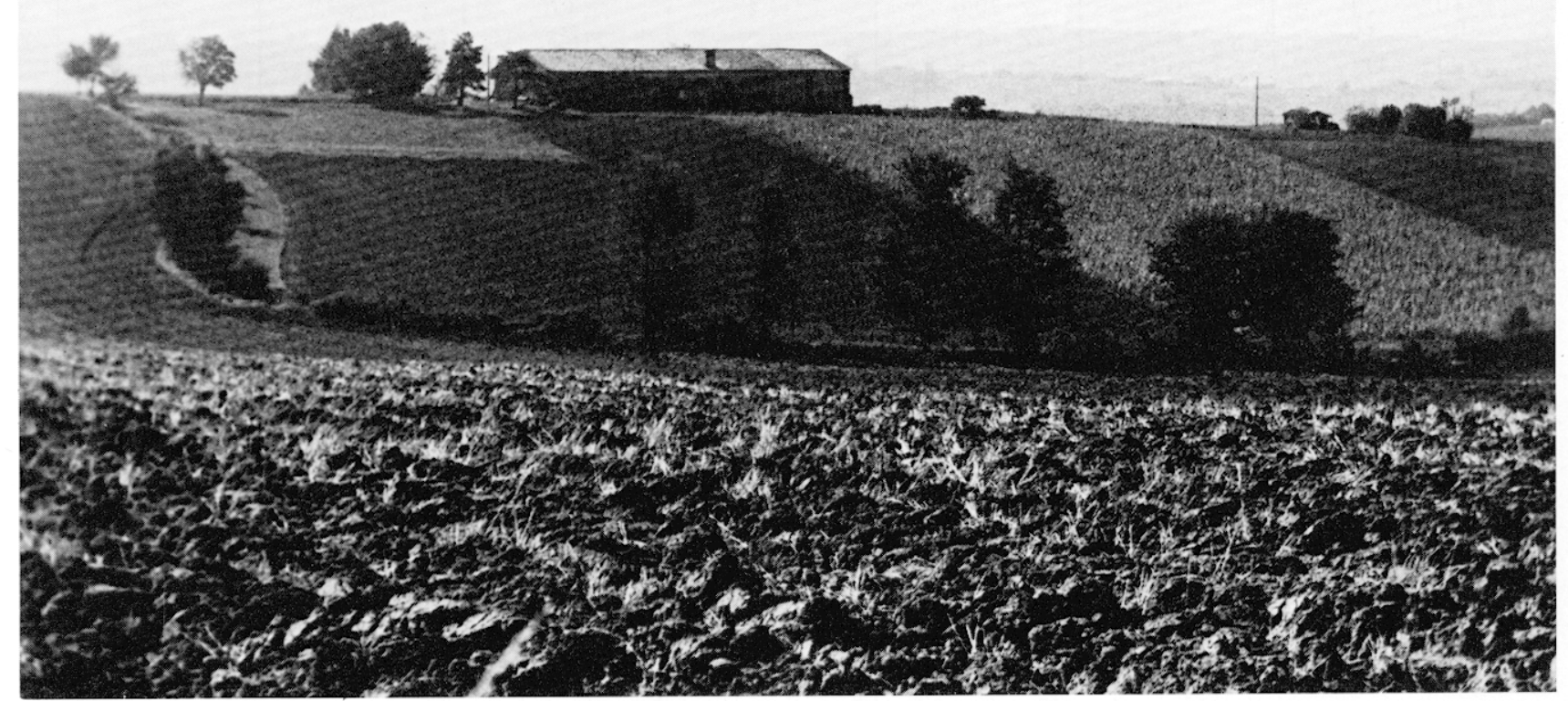

Photo 2 - Le "plateau" molassique lauragais, aujourd'hui réduit à de longues crêtes d'interfluves.

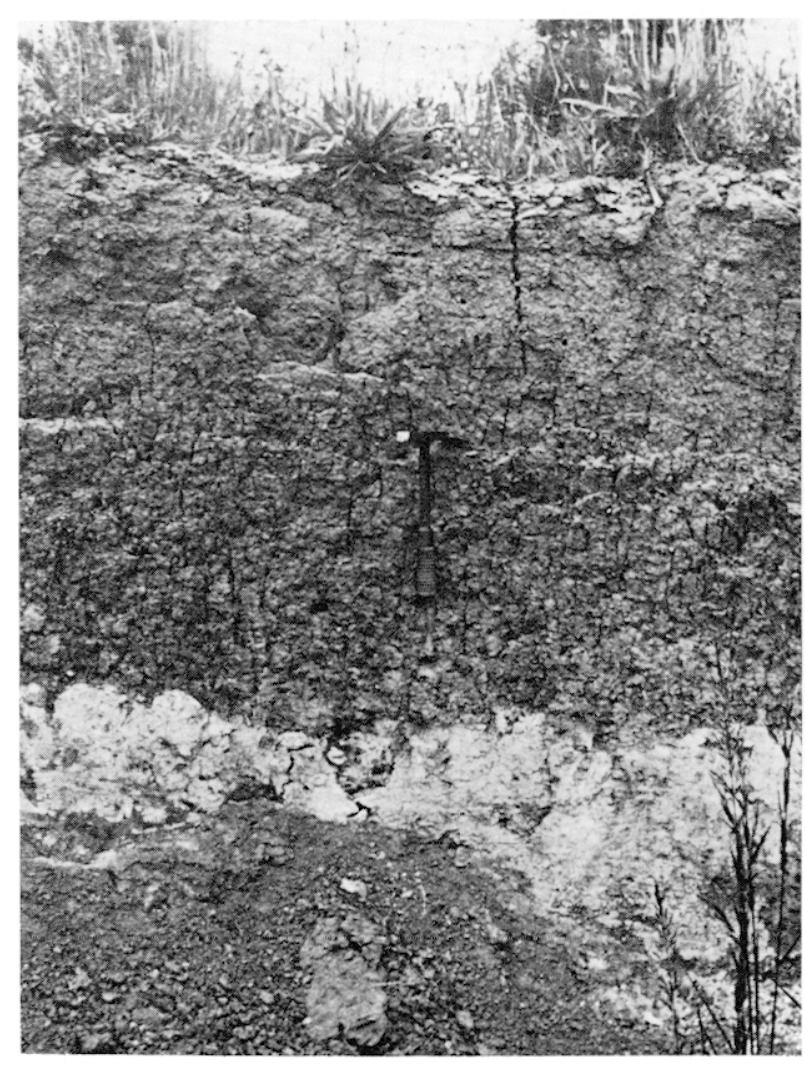

Photo 3 - Sol brun lessivé à accumulation calcaire des éléments de plateau molassiques. rulents et des faciès concrétionnés. Ces derniers (nodules pleins ou creux, esquisses de poupées), qui présentent le plus souvent un état de délabrement avancé, évoquent imparfaitement ceux du loess (arrêt $\mathrm{n}^{\circ}$ 5). Comme pour le loess, ces concrétionnements traduisent peut-être également l'influence de la phase relativement sèche et froide de la fin du Würm, au cours duquel se sont mis en place les placages éoliens. De même, dans et surtout sous l'horizon d'accumulation calcaire, la redistribution du carbonate sous forme de minces lits pulvérulents subhorizontaux, lesquels marquent généralement le sommet de la molasse (photo 4), pourrait résulter aussi d'une ségrégation liée au froid et/ou à la sécheresse de la fin du Würm (A. Cavaillé 1969a, M. Mouline et al., 1969).

Tout ceci invite à penser que le dernier façonnement effectif de ces lambeaux de plateau remonte à une période relativement récente, Riss au plus tôt et plus probablement à la $1^{\text {ère }}$ partie du Würm. Là tout au moins où l'armature de bancs calcaires est négligeable, l'ensemble des éléments qui constituent aujourd'hui la haute surface démantelée du Terrefort s'est donc sans doute abaissé de quelques dizaines de mètres depuis la fin du Tertiaire. Ceci explique que, caillouteuses et plus perméables donc plus résistantes, les plus hautes nappes alluviales garonnaises et ariégeoises occupent une position culminante dans le pay- 
sage molassique, comme on pourra s'en rendre compte au cours de la $2^{\mathrm{e}}$ journée ( $F$. Taillefer 1948, 1951. J. Hubschman 1972a).

\section{C - Les placages loessiques de la vallée de l'Hers mort}

Dans le piémont garonnais, les loess sont rares et surtout très discontinus. D'épaisseur variable (du mètre à la dizaine de mètres exceptionnellement), ils se présentent toujours sous forme de placages d'extension fort limitée : de quelques dizaines de $\mathrm{m}^{2}$ à un hectare. Ces formations sont généralement situées soit au pied des versants molassiques qui bordent à l'ouest les vallées du Terrefort (celle de l'Hers mort tout particulièrement), soit au bas des talus de terrasses, beaucoup plus rarement à l'intérieur même du Terrefort (cf. arrêt $\mathrm{n}^{\circ}$ 2). Les placages qui, au sud-est de Toulouse, jalonnent le versant gauche de la vallée de l'Hers mort sont connus de longue date (G. Astre, 1938, 1956, 1972). Sur les feuilles de Cazères et Saverdun de la carte géologique au $50000^{\mathrm{e}}$, A. Cavaillé a cartographié les petits gisements qui, en rive gauche de l'Ariège, coiffent çà et là le pied du talus molassique de sa terrasse mindélienne. D'autres stations ont été reconnues depuis (fig. 3), notamment aux confluences du Volp et de l'Arize avec la Garonne et au bas de certains talus des terrasses garonnaises (L. Méroc 1969 , J. Hubschman 1972b, 1975a).

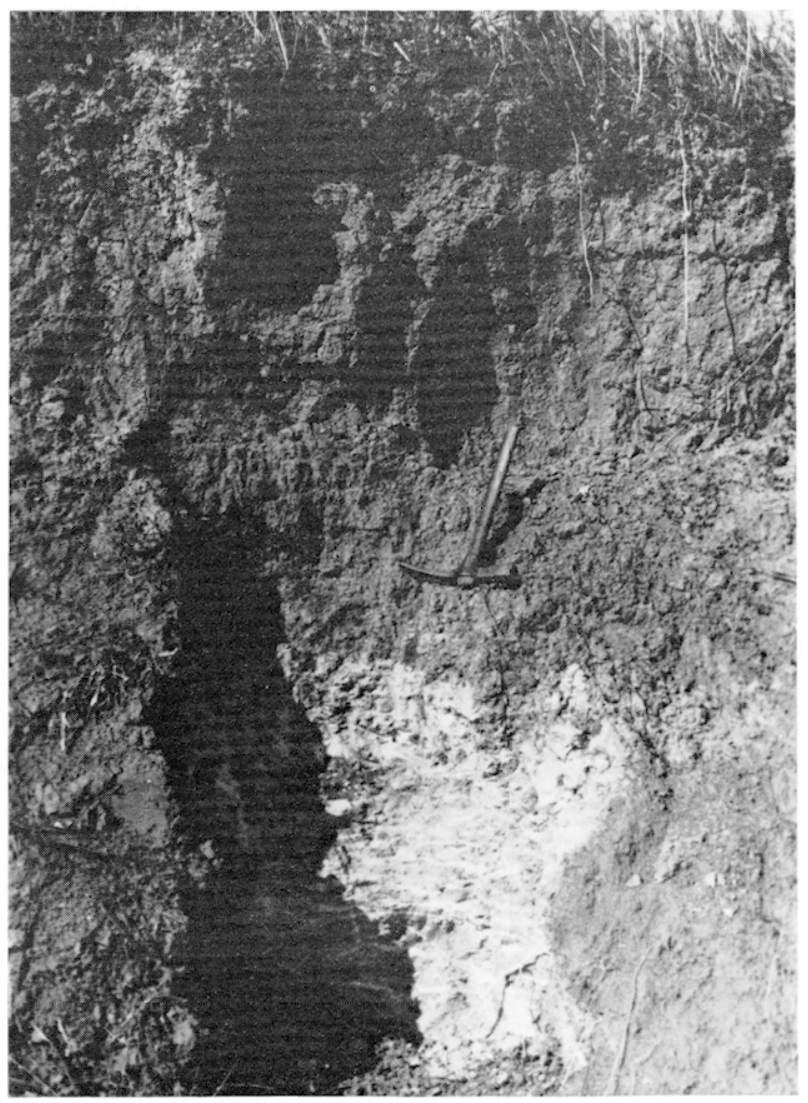

Photo 4 - Lits horizontaux de calcite au sommet de la molasse, sous un profil de sol brun lessivé.

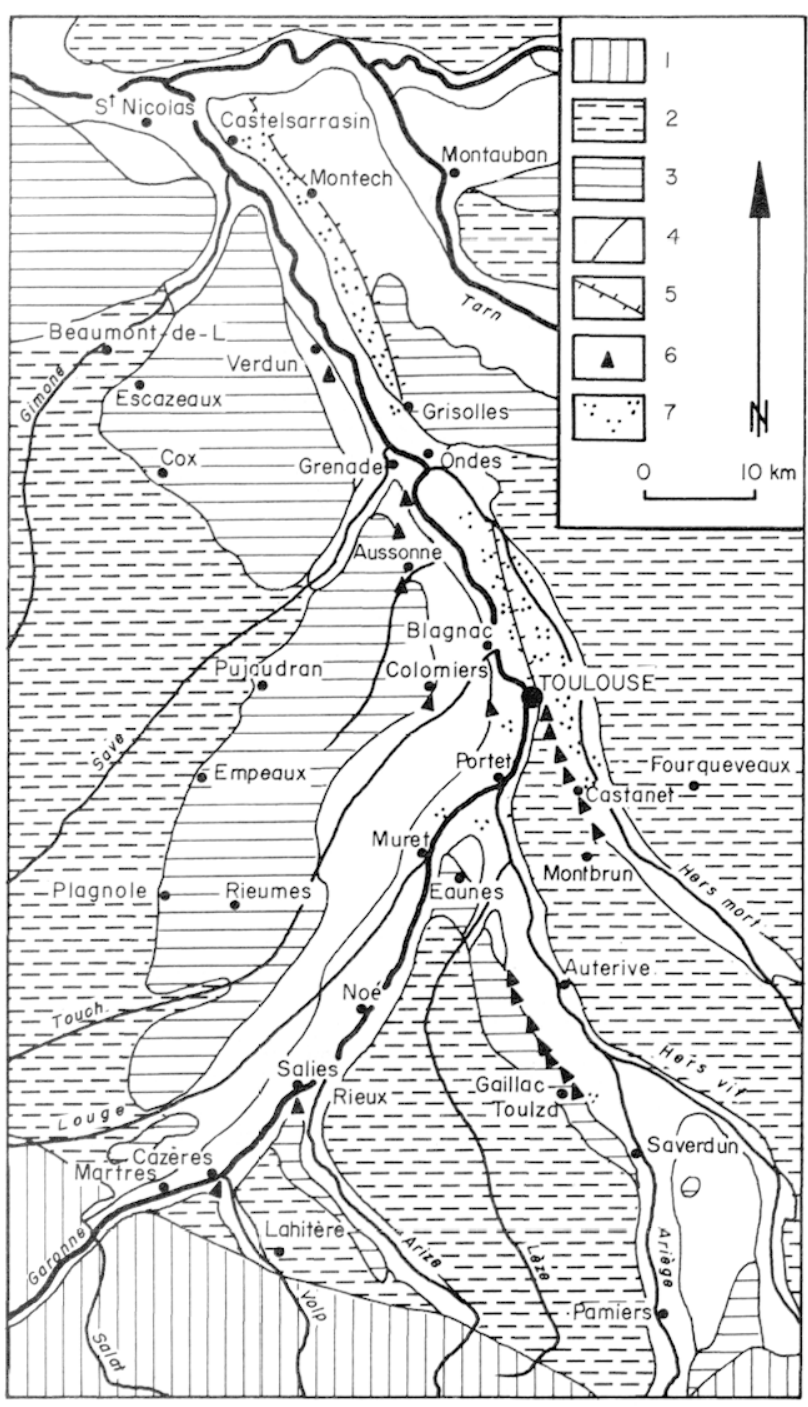

Fig. 3 - Esquisse schématique de la répartition des formations loessiques et des limons éolisés dans l'avant-pays garonnais et ariégeois.

1. Bordure pyrénéenne 2. Molasse 3. Nappes culminantes, hautes et moyennes terrasses 4 . Basses terrasses et leurs talus bordiers, basses plaines et lits majeurs 5 . Talus du palier supérieur de la basse plaine garonnaise à l'aval de Toulouse et du palier inférieur du plateau de Lavilledieu-Montech 6. Placages loessiques 7. Principaux secteurs éolisés des basses plaines.

Le gisement de Pompertuzat (arrêt $n^{\circ} 5$ ) est précisément le plus vaste et l'un des plus épais parmi les placages qui bordent la vallée de l'Hers mort. A une vingtaine de mètres au-dessus de la vallée et en contrebas du vieux village, le dépôt éolien recouvre un bas versant molassique d'interfluve entre deux petits vallons (fig. 4). A la coupe proprement dite, le loess, épais de 6 à 8 mètres, repose sur le substrat molassique. On peut $\mathrm{y}$ distinguer 7 principaux faciès sédimentologiques ou pédologiques (y compris celui de la molasse), d'inégale importance et généralement séparés par des discontinuités plus ou moins clairement exprimées (fig. 5). 


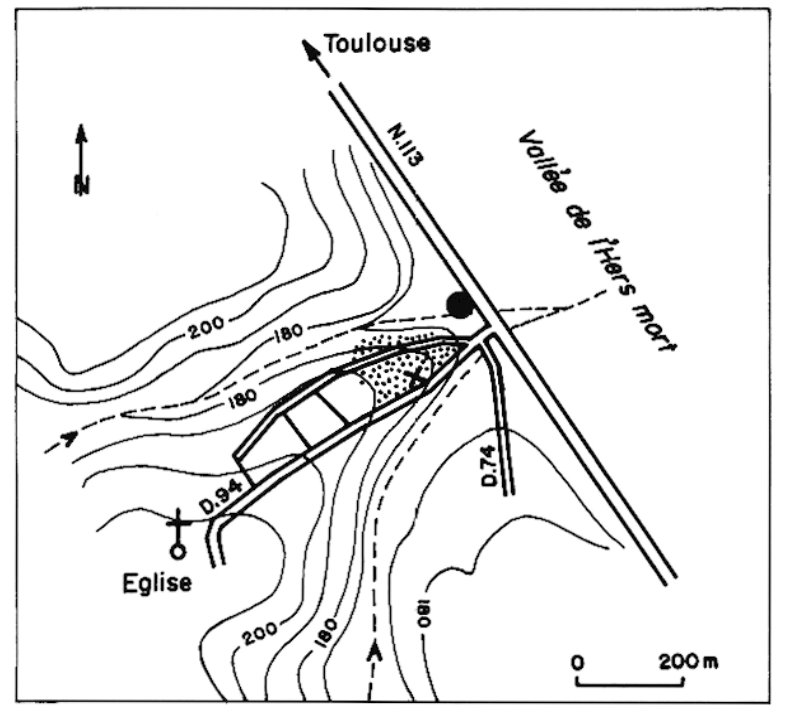

Fig. 4 - Situation du loess de Pompertuzat. En pointillé est figurée l'extension du loess. La croix $\mathrm{x}$ indique le profil étudié.

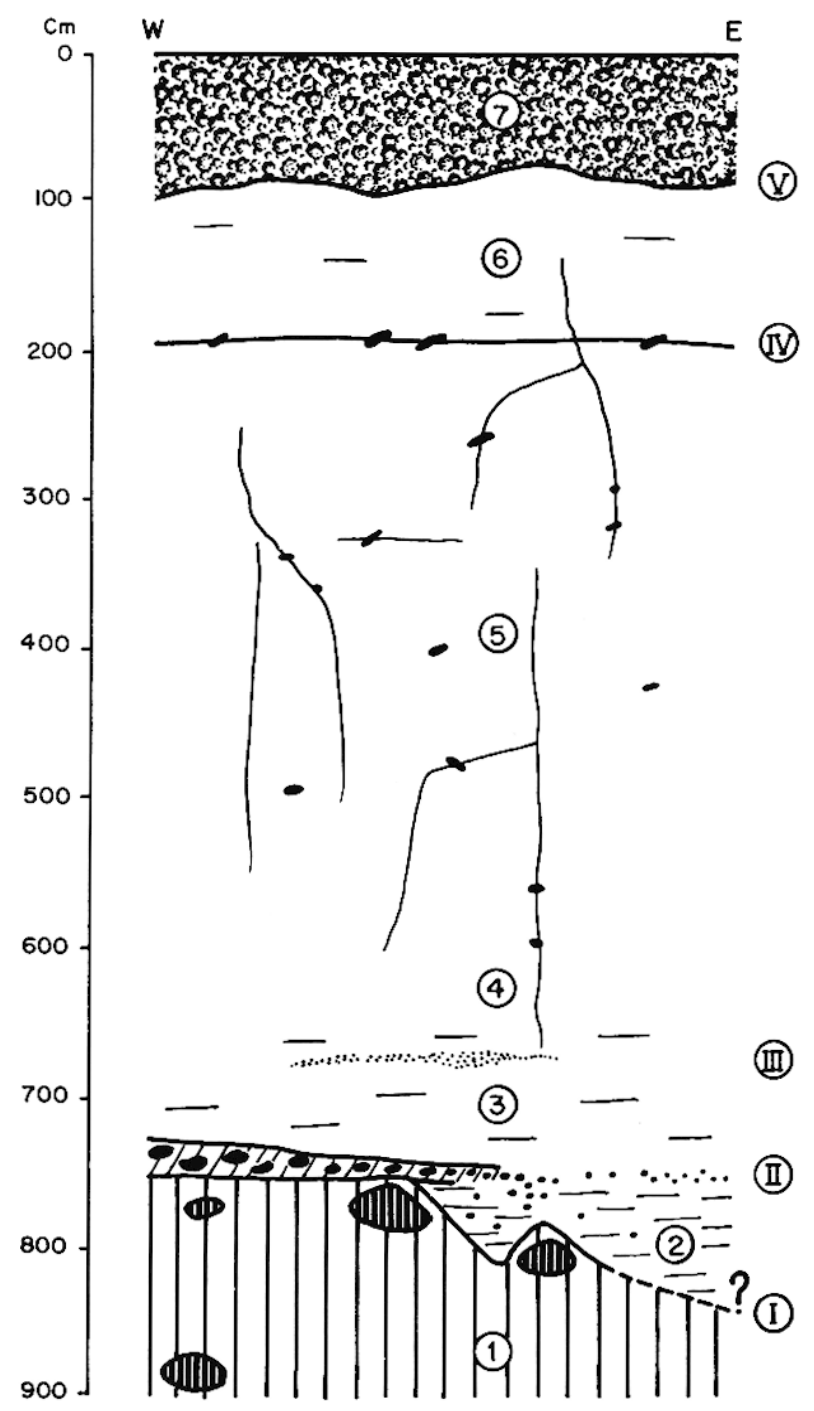

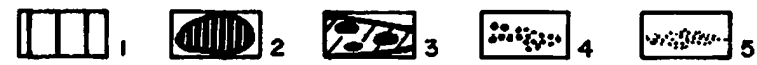
Ex $\quad$ E, (1) 9 (1) 10
- Le sommet de la série molassique (faciès 1 ) est formé d'un matériau argilo-sableux, à éléments gréso. calcaires consolidés par endroit. La distribution des sables est celle, assez classique, de la molasse argilocalcaire (courbe m, fig. 6).

- La base de la formation loessique (faciès 2) ravine la molasse par poches (discontinuité 1). Le matériel, nettement quoiqu'inégalement lité, est relativement riche en sables grossiers, avec par endroit quelques gravillons quartzeux et des concrétions calcaires ou débris de molasse. La distribution des sables (courbes 1 et 3, fig. 6) signale un tri médiocre, sauf au centre de la poche principale à l'est (courbe 2, fig 6) dont l'allure évoque un peu le faciès 5 .

- Le faciès 3 repose, à l'extrémité ouest de la coupe, sur un horizon encroûté et cimenté par le calcaire (discontinuité II), qui scelle ici le sommet de la molasse. Cet horizon induré est formé de débris de molasse et de concrétions calcaires, parfois de galets siliceux (origine molassique?). Vers l'est, cette formation s'amincit, la taille des éléments diminue, la cimentation disparaît peu à peu : on passe alors à une simple assise d'éléments figurés, plus ou moins épaisse, ou à un lit sableux plus sombre, par lequel débute à cet endroit le faciès 3 . Ce dernier est encore nettement lité, mais le tri granulométrique est cependant déjà mieux assuré que dans le faciès précédent (courbes 4 et 5 , fig. 7).

- Du faciès 3 au faciès 4 , la discontinuité III ne se marque véritablement qu'au centre de la coupe, sous forme d'un mince lit plus sableux. Le faciès 4 , légèrement lité, surtout à la base, est un peu plus sombre que le faciès 3 . Il offre des caractères de distribution des sables analogues, malgré une richesse plus marquée en sables fins et grossiers (courbe 6, fig. 7). Le faciès 4 passe très progressivement et sans rupture apparente au faciès 5 ci-dessous.

- Le loess typique est massif, jaune livide, sans litage, à grandes fissures plus ou moins orthogonales qui comportent par place des poupées calcaires. Il constitue le faciès 5 , de loin le plus épais $(4$ à $5 \mathrm{~m})$. Les courbes cumulatives (courbes $1,2,3,4$ et 5 , fig. 8) sont très redressées et régulières, traduisant un tri granulométrique bien assuré. Plus de $80 \%$ des sables rassemblent ainsi les particules comprises entre $40.10^{-3}$ $\mathrm{mm}$ et $120.10^{-3} \mathrm{~mm}$.

Fig. 5 - Le loess de Pompertuzat. 1. Molasse stampienne bigarrée, argilo-sableuse 2. Eléments gréso-calcaires de la molasse. 3. Formation encroûtée à débris de molasse et de grès calcaire 4. Passage latéral de l'encroûtement à des éléments grossiers libres dans une matrice plus sableuse 5 . Lit à tendance sableuse 6. Fissures et poupées ou nodules du loess 7. Litage des faciès (d'autant plus net que le figuré est plus dense) 8. Lehm superficiel très organique et remanié ("terre végétale") 9. Principaux faciès sédimentologiques 10. Principales discontinuités. 


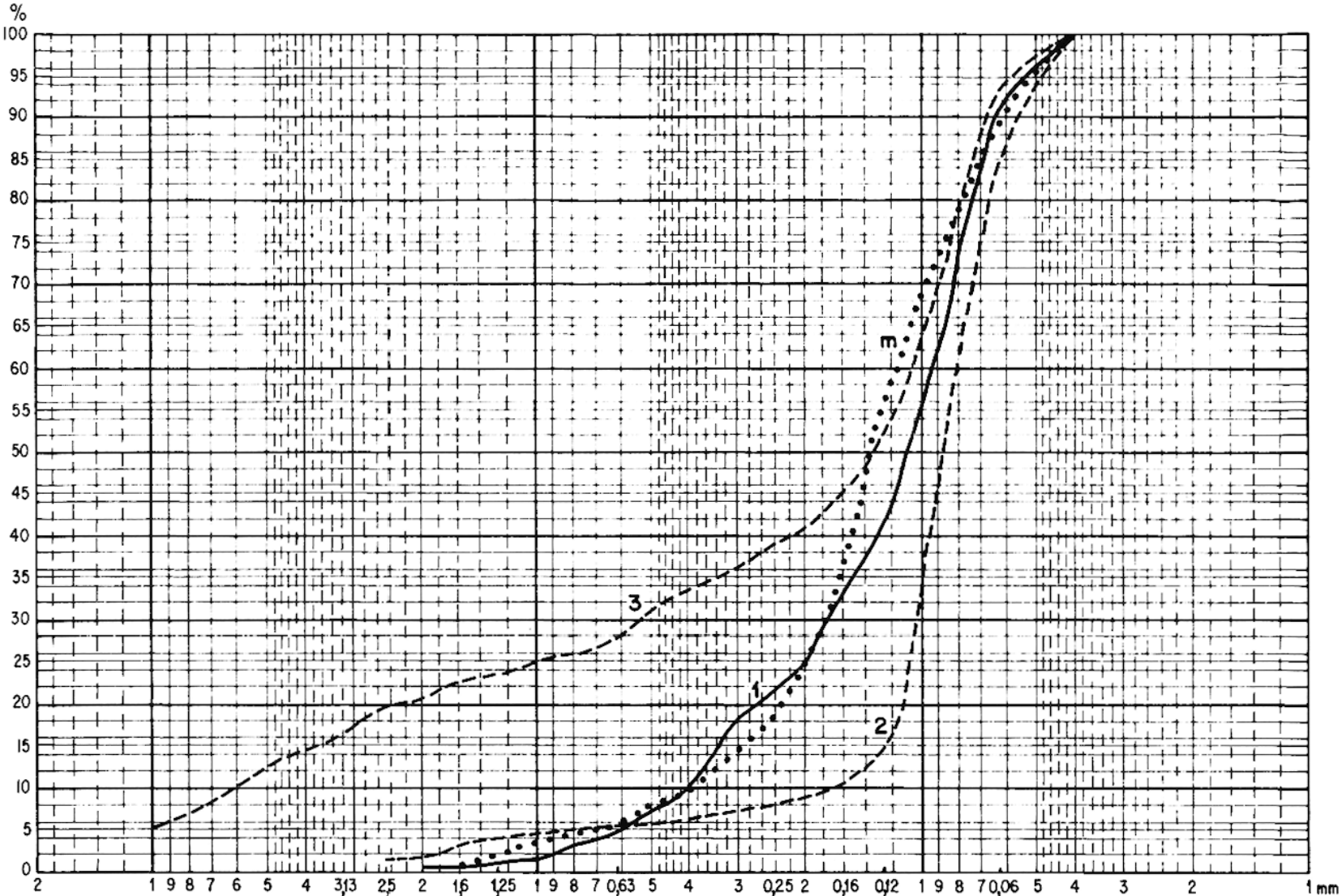

Fig. 6 - Distribution fréquentielle des sables (loess de Pompertuzat).

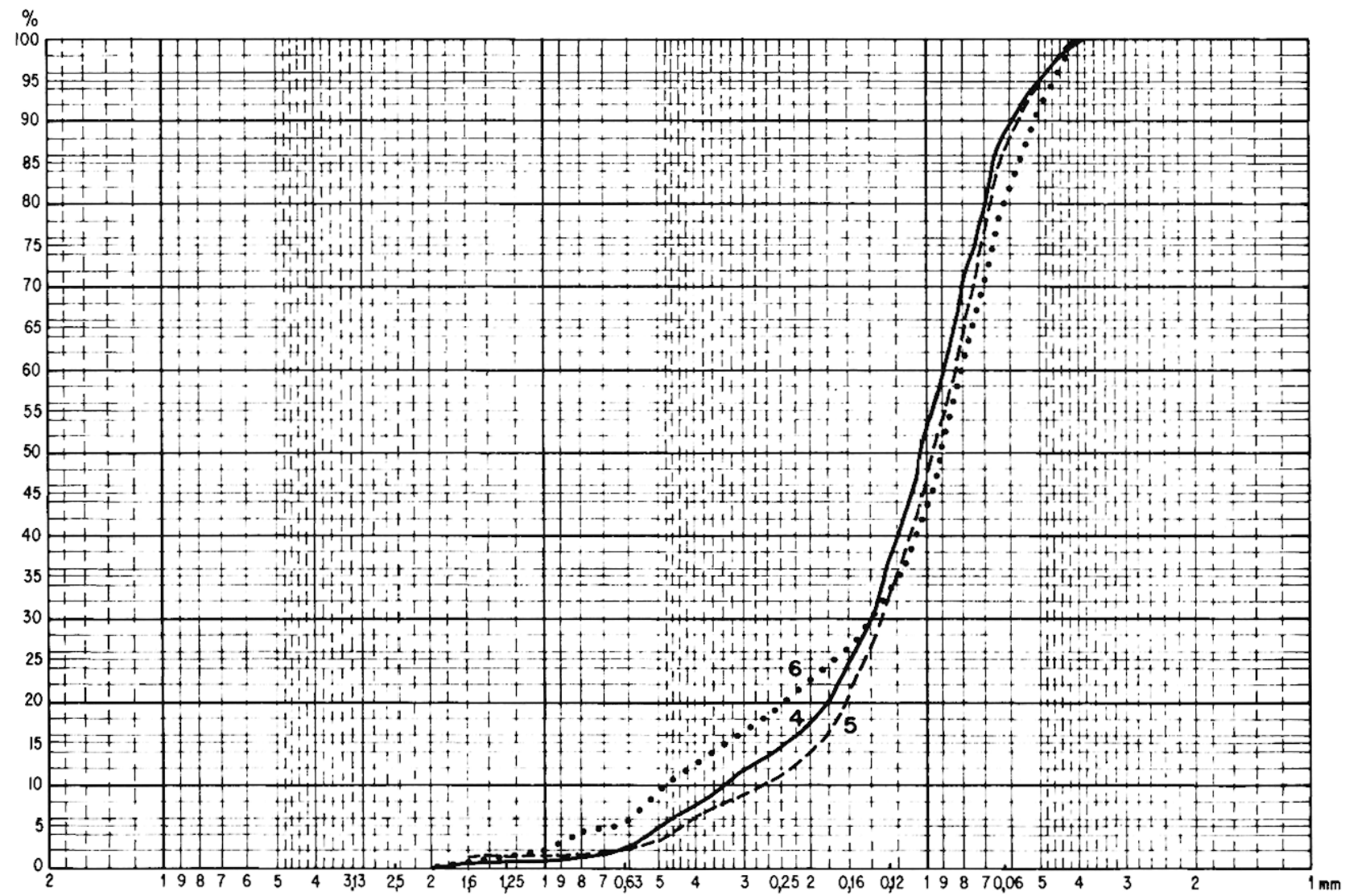

Fig. 7 - Distribution fréquentielle des sables (loess de Pompertuzat-suite). 


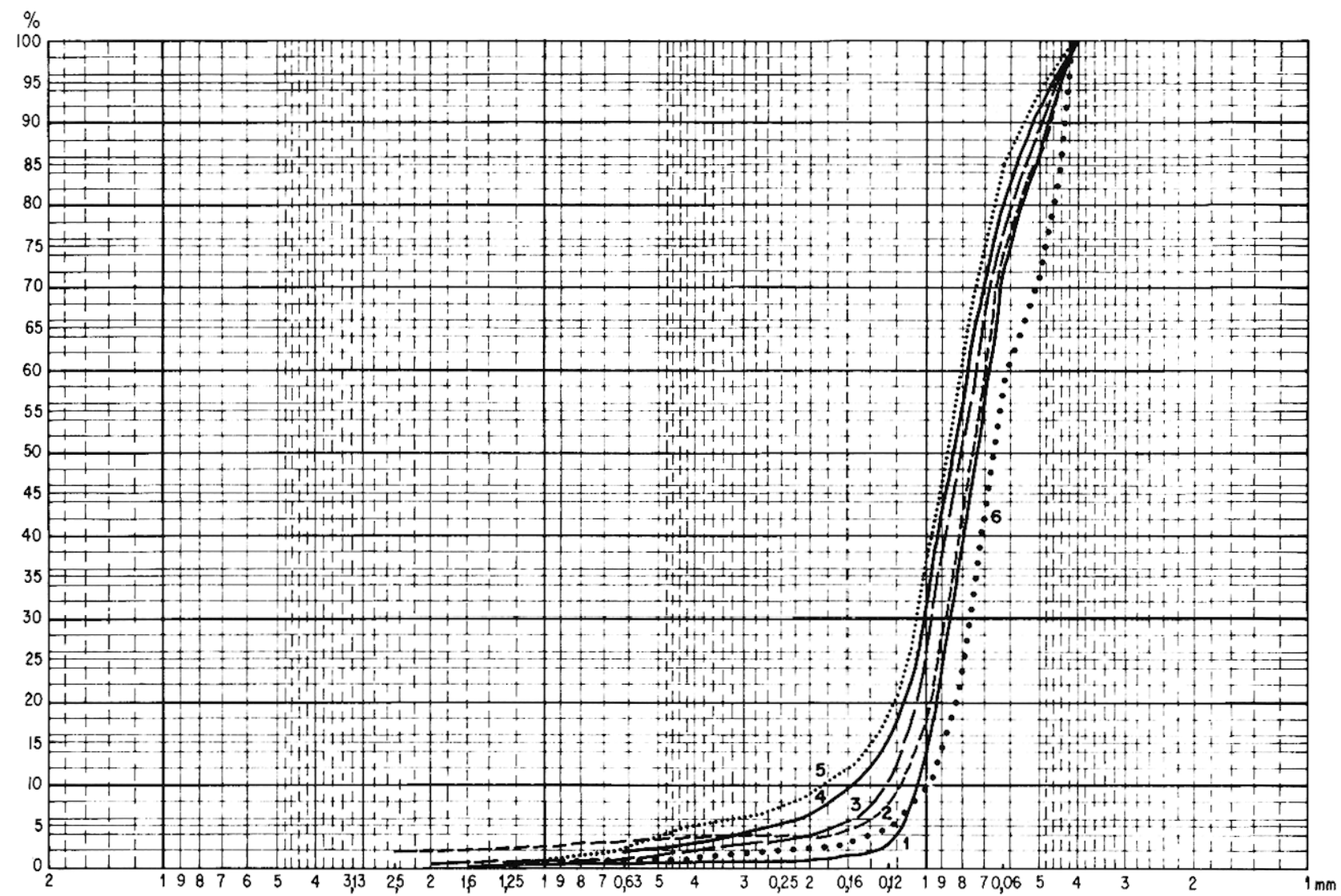

Fig. 8 - Distribution fréquentielle des sables (loess de Pompertuzat-suite).

$\Lambda$ u-dessus de la discontinuité IV, simple fissure subhorizontale ponctuée çà et là de quelques poupées calcaires, le faciès 6 se marque par un très discret litage. De fait, la distribution des sables, quoique révélatrice d'un excellent tri, est moins régulière que dans le faciès précédent (courbe 6 , figure 8 ).

- La couche superficielle, d'épaisseur très variable en réalité, peut être assimilée à un lehm très organique, très remanié et pénétré par un dense réseau de racines. En raison de ce remaniement important (bordure de talus routier, ancienne carrière), on n'observe plus guère le profil brun lessivé caractéristique des lehms postglaciaires qui ont pu être étudiés ailleurs (J. Hubschman, 1975b).

La composition chimique et minéralogique de ces loess n'appelle pas de commentaire particulier, sinon à propos de l'identité minéralogique (minéraux lourds et minéraux argileux surtout) entre molasse et loess. Quelle qu'ait été la direction des vents dominants au moment du dépôt, de secteur ouest (D. Faucher 1931, H. Enhalbert 1960) ou, plus vraisemblablement, de secteur est (G. Astre 1972), il est clair que les poussières éoliennes tirent leur origine des assises molassiques (ou des sols peu évolués qui en dérivaient). Il est également clair qu'elles ne se sont guère déposées très loin de leur point de départ, probablement à quelques centaines de mètres dans nombre de cas. Parfois, l'accumulation s'est produite à proximité immédiate de l'affleurement molassique lui-même: ainsi en est-il pour les placages des talus molassiques des terrasses (J. Hubschman 1975a).

L'éolisation du matériel ressort déjà des courbes cumulatives des sables, tout au moins pour le faciès 5 . Elle apparaît encore mieux à l'observation exoscopique des grains de quartz (J. Hubschman et L. Le Ribault 1972). Les photos 5 et 6 (grossissements respectifs 1000 et 10000 , microscopie électronique à balayage) montrent des grains issus d'une accumulation loessique typique, analogue au faciès 5 de Pompertuzat et provenant d'un dépôt voisin (Toulouse-Bcllevue). La morphologie de surface des quartz est très symptomatique : les arêtes des grains exposées aux chocs sont entaillées en " $V$ " bien marqués (photo 5 et à bords anguleux (photo 6 ; les faces planes portent des traces de percussions nombreuses mais peu profondes (photo 6). L'éolisation est "fraîche" et représente à coup sûr le dernier stade d'évolution des grains.

L'âge des loess a pu être rapporté au Würm supérieur par J.J. Puysségur (in A. Cavaillé, 1969a), grâce aux paléobiocénoses de Gastéropodes, notamment: Vitrina (Crystallus) pseudohydatina Bourg., Fruticola hispida L., Helicella striata Mull., Clausilia parvula Stud., Succinea valcourtiana Bourg. etc. (G. Astre 1952). De fait, une mesure d'âge au radiocarbone, 


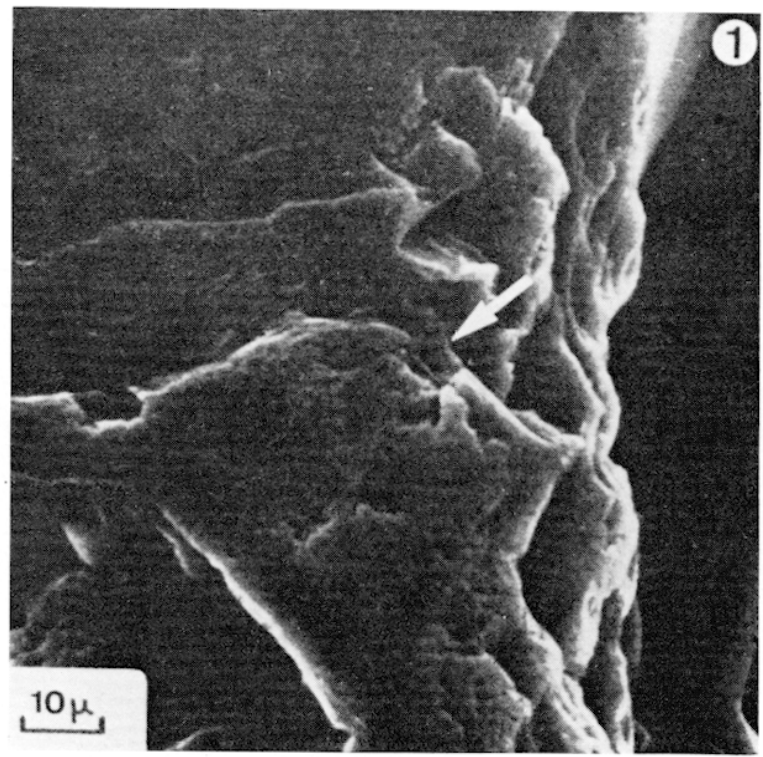

Photo 5 - Eolisation des quartz du loess. La flêche indique la localisation de la photo 6 .

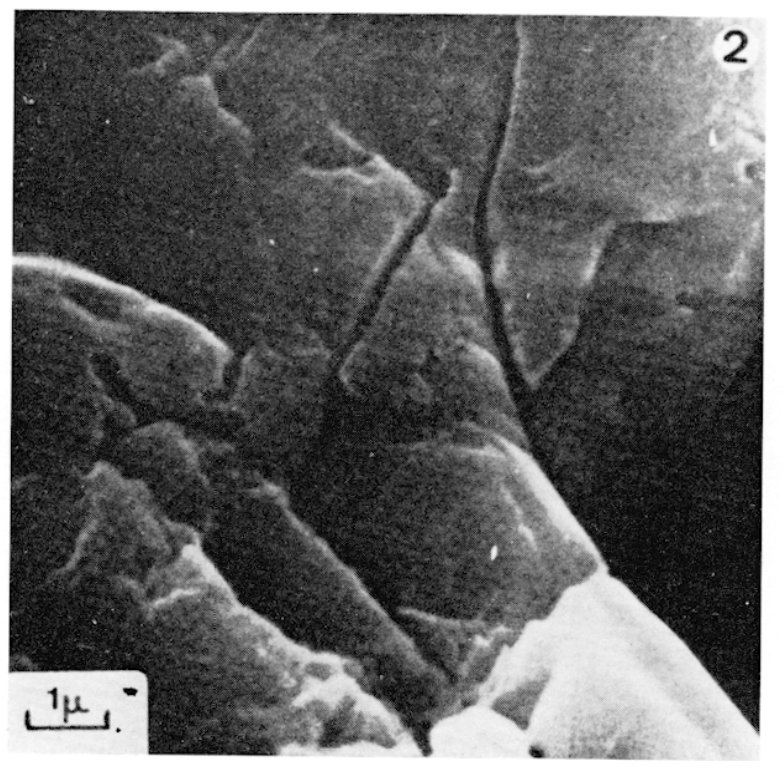

Photo 6 - Eolisation des quartz du loess (grossissement local de la photo 5 ).

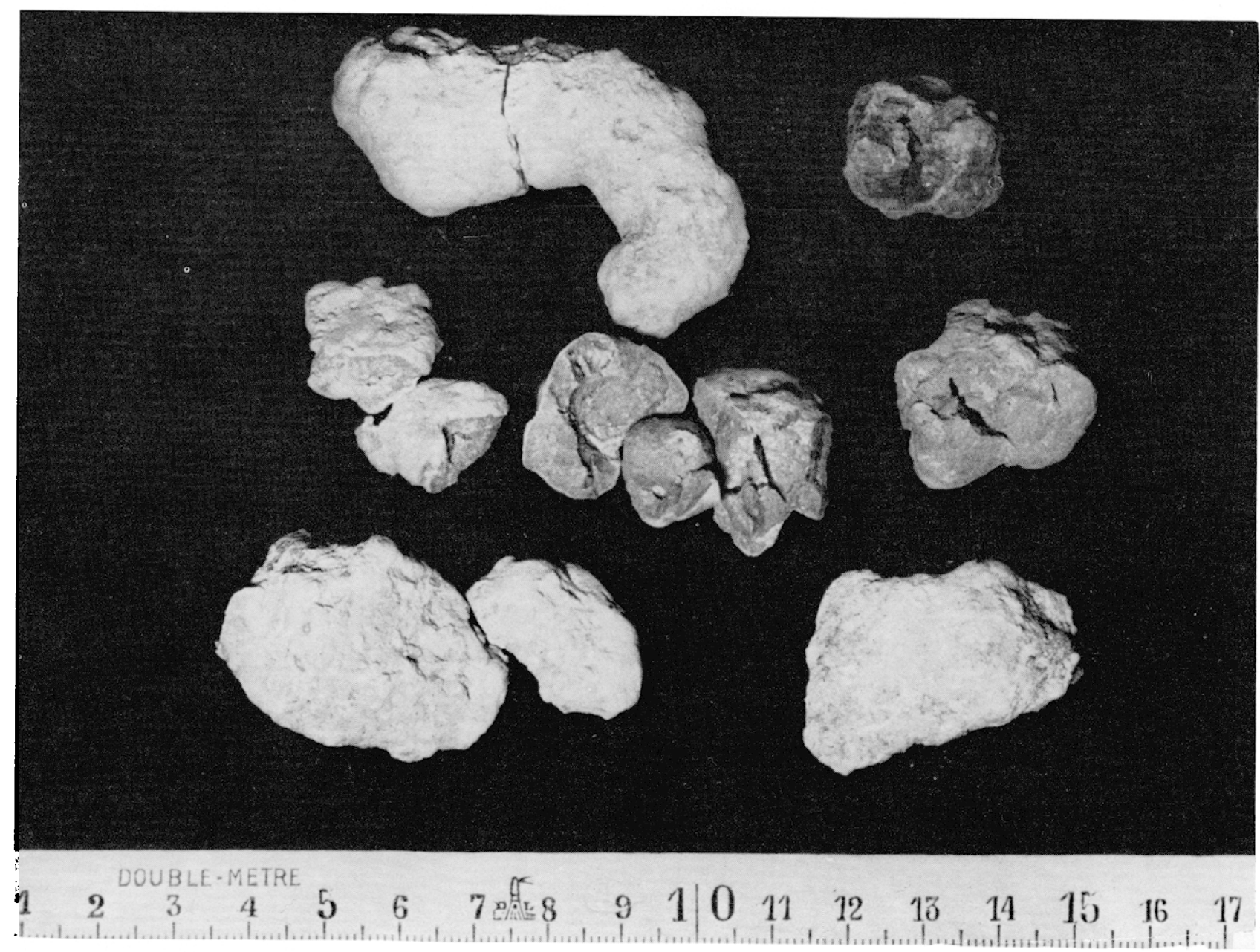

Photo 7 -- Les concrétionnements calcaires du loess. 
effectuée sur les coquilles de Gastéropodes fossiles de la base du faciès 5 , a donné $20900 \pm 570$ ans B.P. (1) (J. Hubschman 1973). Cette datation cadre assez bien avec l'estimation précédente. Ce résultat devrait cependant faire l'objet d'un contrôle en opérant à nouveau non plus sur les carbonates des coquilles mais à partir du carbone organique résiduel du loess (2).

L'approche paléogéographique et paléoécologique du loess ne peut guère être qu'esquissée pour le moment. Les couches inférieures du gisement trahissent l'action du ruissellement (faciès 2 et 3 surtout). Le faciès 5 , en

(1) Mesure faite sous la direction de Mme G. Delibrias, Laboratoire du Radiocarbone du CNRS, Gif-sur-Yvette.

(2) Suggestion de J. Evin, Laboratoire du Radiocarbone, Université Claude-Bernard, Lyon. revanche, est purement éolien. Ceci pourrait traduire, sous toutes réserves, le passage d'un épisode würmien encore relativement tempéré et humide à une phase véritablement propice à l'éolisation, plutôt sèche et froide et peu biotique, ce que l'affinité froide et/ou sèche des Gastéropodes traduit également (1). Dans cette optique, les concrétionnements calcaires du locss, quoique souvent tributaires de la fissuration des dépôts et de l'hydrodynamique qui en résulte, pourraient aussi refléter les forts contrastes d'humidité et de dessication propres à cette phase xérique, comme l'indiquent peutêtre la cristallisation très poussée et les fentes de retrait des concrétions (photo 7).

(1) L'analyse palynologique de G. Jalut est en cours. L'ensemble des données sédimentologiques, géochimiques et palynologiques sera repris dans une publication ultérieure.

Bibliographie

(Voir p. 214). 